\title{
Mehr Qualität und Patientensicherheit - Managed Care als Schlüsselfaktor
}

\section{Peter Berchtold}

Präsident Forum Managed Care*

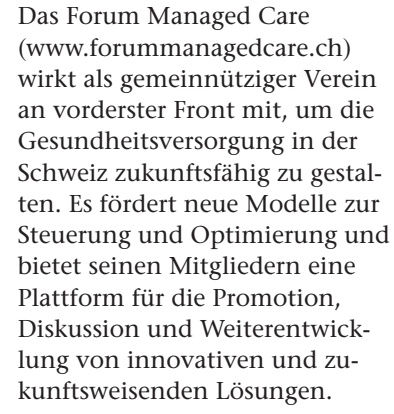

Das Forum Managed Care (www.forummanagedcare.ch) wirkt als gemeinnütziger Verein an vorderster Front mit, um die Gesundheitsversorgung in der Schweiz zukunftsfähig zu gestalten. Es fördert neue Modelle zur Steuerung und Optimierung und bietet seinen Mitgliedern eine Plattform für die Promotion, Diskussion und Weiterentwicklung von innovativen und zukunftsweisenden Lösungen.

Korrespondenz:

MKR Consulting AG

Marketing- und Kommunikationsberatung

Steinerstrasse 37

CH-3006 Bern

Tel. 0313504050

Fax 0313504051

info@mkr.ch

www.mkr.ch
Im konkreten Fall ist nur das Beste gut genug! Die uneingeschränkte Zugänglichkeit, vor allem aber die Qualität der gebotenen Behandlungen und das zu erwartende «Outcome» prägen die Erwartungen der Schweizer Patientinnen und Patienten an unser Gesundheitswesen. Zudem werden immer deutlicher Fragen zur Patientensicherheit gestellt. Gemäss den aktuellen Meinungsumfragen sind das die Themen, mit denen sich Versicherte wie auch Patientinnen und Patienten in erster Linie beschäftigen. Kosteneinsparungen, höhere Selbstbehalte oder Prämienreduktionen scheinen erst in Funktion zum gebotenen Nutzen als wichtig erachtet zu werden. Mit zunehmender Vielfalt und Komplexität von Behandlung und Betreuung - besonders bei chronischen Krankheiten - sind die Behandlungsqualität und die Patientensicherheit direkt vom Zusammenspiel aller Beteiligten abhängig. Es reicht daher heute nicht mehr, wenn einzelne Untersuchungen, Behandlungen und Betreuungsmassnahmen qualitativ hochstehend sind. Was zählt, sind gleichzeitig die Qualität im Zusammenspiel und das Gesamtresultat.

\section{Fehler entstehen an Bruchstellen}

Qualität und Sicherheit in Behandlung und Betreuung sind auch zentrale Ziele der Leistungserbringenden, ob in Einzelpraxis, Ärztenetz, Spital, Spitex usw. Ebendiese Qualität und Sicherheit entstehen aber nicht mehr (nur) an «lokaler» Stelle, sondern (auch) im Rahmen eines funktionierenden Zusammenwirkens beteiligter Akteure. Ein funktionierendes Zusammenwirken ist offensichtlich herausforderungsreicher, je mehr Personen und Institutionen am Behandlungs- und Betreuungsprozess beteiligt sind. Darüber hinaus wird heute das Zusammenwirken unter anderem durch die zunehmende Ökonomisierung der Medizin bedrängt, denn viele ökonomische Anreize entfalten zwar für einzelne Akteure betriebswirtschaftlich erwünschte Wirkungen, leider aber auch medizinisch unerwünschte Nebenwirkungen. Beispielhaft dafür ist die in Deutschland im Rahmen der DRG-Einführung zu beobachtende Zergliederung der Patientenprozesse in (ökonomisch) definierte Teile, die ihrerseits zu neuen Informationsbrüchen und Pannen führen können.

\section{Managed care: facteur clé pour accroître la qualité et la sécurité des patients}

Le symposium du Forum du Managed Care (www.forummanagedcare.ch) qui sera tenu le 6 juin 2007 aura pour thème le managed care, facteur clé pour accroître la qualité et la sécurité des patients. Des experts suisses et étrangers apporteront de nouvelles idées et présenteront leurs expériences et leurs solutions. L'après-midi, les participants auront l'occasion de discuter dans des ateliers interactifs sur un choix de projets et d'expériences et de chercher ensemble des solutions. En parallèle, différents exposés donneront un aperçu des projets en train d'être réalisés en Suisse et les participants pourront à nouveau acquérir des connaissances générales en managed care dans le cadre de six ateliers de formation.

\section{Wirkungsvolle Verknüpfungsstrategien ...} Nicht nur im Gesundheits-, sondern auch in anderen Bereichen stehen Organisationen vor der Herausforderung, dass zunehmende Spezialisierungen und komplexere Technologien Leistungsqualität bedrohen und Fehleranfälligkeit erhöhen können: Die sogenannten «high reliability organisations» (HROs) - Atomkraftwerke, Flugzeugträger u.a. - sind solche Organisationen, die eine hohe Zuverlässigkeit gewährleisten müssen trotz ihrer inhärenten Risiken, Menschen schwer zu schaden. In vielen Organisationen werden Qualität und Sicherheit durch Standardisierung und Vereinheitlichung der operativen Abläufe erreicht. Hierarchische Kontrolle im Sinne eines klassischen Managements hat dabei die Aufgabe, Abweichungen von definierten Standards zu minimieren bzw. zu korrigieren, um dadurch Fehler zu verhindern.

In Organisationen mit hochkomplexen Aufgaben, in Prozessen mit einer Vielzahl unterschiedlicher Beteiligter und nicht zuletzt dort, 
wo das Kerngeschäft in den Händen professioneller Experten liegt - wie das in Spitälern und anderen Gesundheitseinrichtungen der Fall ist -, greifen ein klassischer Managementansatz und hierarchische Kontrolle zu kurz: Auch noch soviel Standardisierung, Leistungsindikatoren, Benchmarking und wissenschaftliche Evidenz in der klinischen Praxis werden letztlich nur unbefriedigende Resultate bezüglich Qualität und Sicherheit bewirken können, mehr noch, sie können bei den Beteiligten ein gefährliches falsches Gefühl von Verlässlichkeit und Sicherheit erzeugen. Daher ist im Gesundheitsbereich ein integrativer Managementansatz zu verfolgen, der

- (neben Standardisierung, EBM und Leistungsindikatoren) die prekären Beziehungen zwischen den differenzierten Gruppen sowohl im Spital (Ärzte, Pflegende, Manager, Patienten usw.) wie auch im Gesamtsystem (Leistungserbringer, Versicherer, Staat, Versicherte usw.) berücksichtigt und diesen Rechnung tragen kann;

- dem Verständnis unterschiedlicher (berufs-) kultureller Werte und Haltungen sowie gegenseitigem Respekt grundlegende Beachtung schenkt;

- Kooperation und kontinuierliches Lernen über traditionelle Grenzen hinweg fördert.

\section{... und Managed Care als Schlüssselfaktor ...}

In Analogie zum integrativen Managementansatz geht ein modernes Verständnis von Managed Care ebenso über die traditionellen Inhalte wie Gatekeeping, Ärztenetze und Budgetmitver- antwortung hinaus und folgt einem systemischen Ansatz der Integration und der verstärkten Verkoppelung der unterschiedlichen Akteure mit dem Ziel, deren Zusammenwirken weiter zu optimieren und damit Qualität der Behandlung und Sicherheit der Patienten zu stärken. Das müsste eigentlich auch jene Leistungserbringenden von Managed Care überzeugen, die dazu bisher auf Distanz blieben. Und auch die Tatsache, dass Managed Care in der Schweiz zu wesentlichen Teilen von Leistungserbringenden initiiert, aufgebaut und weiterentwickelt wurde und sich Staat und Versicherer (wenn auch nicht konsequent) auf Förderung beschränkt haben. Diese Möglichkeit des Entwickelns «an der Front» und nicht auf staatliches Geheiss oder kontraproduktiven Druck der Finanzierenden hat Wesentliches zur Attraktivität von Managed Care in der Schweiz beigetragen und wird es weiterhin tun.

\section{... am diesjährigen Symposium des Forums Managed Care}

$\mathrm{Zu}$ diesen Themen werden am Symposium am 6. Juni 2007 Experten aus dem In- und Ausland neue Impulse einbringen sowie ihre Lösungsansätze und Erfahrungen vorstellen. Am Nachmittag werden in interaktiven Workshops ausgewählte Konzepte und Erfahrungen zur Diskussion gestellt, um gemeinsam mit interessiertem Fachpublikum nach Lösungen zu suchen. Parallel dazu bieten verschiedene Projektpräsentationen einen aktuellen Überblick über laufende Projekte in der Schweiz und kann in sechs Educational Workshops wiederum allgemeines Managed-Care-Wissen erworben werden.

\section{Mehr Qualität und Patientensicherheit - Managed Care als Schlüsselfaktor}

Datum: 6. Juni 2007, ab 8.30 Uhr geöffnet

Ort: World Trade Center Zürich

Symposiumsbeginn: $9.15 \mathrm{Uhr}$

Symposiumsende: 17.15 Uhr und Apéro, anschliessend (fakultativ) Managed-Care-Networking-Dinner in der alten Giesserei Oerlikon

Kosten: Fr. 480.-, mit Dinner Fr. 580.- (+ MWSt)

Informationen/Anmeldung: www.forummanagedcare.ch 